\title{
Rosen-Morse Kuantum Kuyusunun Safsızlıkla İlgili Doğrusal Olmayan Optik Özellikleri
}

\section{Impurity-Related Nonlinear Optical Properties of Rosen-Morse Quantum Well}

\begin{abstract}
Aslan Türkoğlu ${ }^{1 *}$
Geliş / Received: 31/03/2021

Kabul / Accepted: 13/07/2021

\section{ÖZ}

Bu çalışmada, dış statik elektrik ve manyetik alanın Rosen-Morse kuantum kuyusundaki temel durumla ilişkili hidrojenik donor safsızlık atomunun bağlanma enerjisi üzerindeki etkisi teorik olarak etkin kütle yaklaşımında varyasyonel bir yöntem kullanılarak araştırılmıştır. Bu yapıdaki safsızlıkla ilişkili doğrusal olmayan optik özellikler, kompakt yoğunluklu matris yaklaşımı kullanılarak hesaplanmıştır. Doğrusal olmayan optik özelliklerin sayısal sonuçları, merkezi donor safsızlı̆ı için statik elektrik ve manyetik alanın iki farklı değeri için gelen foton enerjisinin fonksiyonları olarak sunulmuştur. Ayrıca, hidrojenik donorsafsızlığının bağlanma enerjisi, statik elektrik ve manyetik alanın bir fonksiyonu olarak gösterilmiştir. Sonuç olarak, yapının doğrusal olmayan optik özelliklerinin, donor atomunun varllğından ve statik elektrik ve manyetik alanların büyüklüğünden önemli ölçüde etkilendiği bulunmuştur.
\end{abstract}

Anahtar Kelimeler- Kuantum Kuyusu, Safsızlık Bağlanma Enerjisi, Elektrik ve Manyetik Alan

\begin{abstract}
In this present study, the effect of external static electric and magnetic field on the binding energy of hydrogenic donor impurity associated with the ground state in Rosen-Morse quantum well were theoretically investigated by using a variational method in the effective-mass approximation. The impurity-related nonlinear optical properties in this structure were calculated by using the compact-density-matrix approach. The numerical results of nonlinear optical properties were presented as functions of incident photon energy for two different values of the static electric and magnetic field for on-center donor impurity. Moreover, the binding energy of the hydrogenic donor impurity was shown as a function of static electric and magnetic field. As a result, it was found that the nonlinear optical properties of the structure were significantly affected by the impurity atom and the magnitude of static electric and magnetic fields.
\end{abstract}

\section{Keywords- Quantum Well, Impurity Binding Energy, Electric and Magnetic Field}

*Sorumlu yazar iletişim: aslan.turkoglu@gmail.com (https://orcid.org/0000-0002-4260-6217) Bağımsız Araștırmacı, Ankara, Türkiye. 


\section{GíRIŞ}

Metal Organik Kimyasal Buhar Biriktirme (MOCVD) ve Moleküler Işın Epitaksi (MBE) gibi gelişmiş yapay kristal büyütme teknikler vasıtası ile kuantum kuyuları (QWs) gibi yüksek kaliteli düşük boyutlu yariiletken heteroyapıların üretilmesi mümkün hale gelmiştir. Ayrıca, bu büyüme yöntemleri bize kare, parabolik, yarıparabolik, eğimli, basamak-tipi, V-şekilli, ters parabolik, Pösch-Teller, Gaussian, WoodsSaxon, Morse, Tietz-Hua ve Rosen-Morse gibi farklı kuşatma geometrilerine sahip kuantum kuyularını elde etmemizi sağlar [1-11]. Farklı geometrik kuşatma potansiyeline sahip tüm kuantum kuyularında, önemli bir şekilde ortaya çıkan bazı önemli fiziksel özellikler vardır. Bu yapıların en önemli fiziksel özelliklerinden biri, farklı sınırlı alt bant enerji seviyeleri arasındaki elektronik geçişlere dayanan optik özellikleridir. Ayrıca, yarıiletken kuantum kuyularının geometrik şekli elektronun hareketi üzerinde önemli bir kısıtlama etkisine sahiptir ve bu nedenle bu kuantum kuşatma etkisi, bu sistemlerin elektronik yapısını önemli ölçüde etkiler. Farklı sınırlı alt bant enerji seviyeleri bu yapılar içinde güçlü bir şekilde sınırlandırıldığından, taban ve ilk uyarılmış seviyeler arasındaki enerji ayrımı nispeten küçüktür, ancak bu yapılar daha büyük dipol moment matris elemanlarına sahip olurlar. Bu modifikasyon, elektronik durumların ve dolayısıyla bu sistemlerin optoelektronik özelliklerinin değişmesine neden olur. Böylece bu modifikasyonlar sistemlerin daha yüksek rezonans genlikli THz bölgesindeki optik yanitlar vermesine neden olur. Sonuç olarak, lazerler, foto-diyotlar, optik anahtarlar, faz ve elektro soğurma modülatörleri gibi optoelektronik aygıtlar yukarıdaki fiziksel özelliklere sahip bu kuantum kuyularının kullanımıyla üretilebilir [12-15]. Yukarıda özetlenen sonuçlar bize gösteriyor ki, bu yapıların doğrusal olmayan optik özellikleri hakkında detaylı bir çalışma yapılması gerekmektedir.

Kuantum kuyularından üretilen cihazların elektronik ve optik özelliklerini iyileştirmek ve kontrol etmek için safsızlıkların ve dış alanların varlığı ve etkisinin dikkate alınması büyük önem taşımaktadır. Bu nedenle, elektrik, manyetik ve yüksek frekanslı yoğun lazer alanları (ILF) gibi harici alanların, kuantum kuyu yapılarındaki hidrojenik donor safsızlığının bağlanma enerjisi ve ilgili optik spektrum üzerindeki etkisi yoğun bir şekilde araştırılmış ve halen araştırılmaktadır [16-24]. Örneğin; elektrik alan altındaki bir süper örgünün doğrusal ve doğrusal olmayan optik soğurma katsayıları Shi ve Pan [16] tarafından hesaplanmıştır. Yoğun lazer alanının oluşturduğu potansiyelin kuşatılmış enerji durumları üzerindeki etkisi Radu [17] tarafından incelenmiştir. Karimi ve Vafaei [18] yüksek gerilimli $\operatorname{In}_{x} \mathrm{Ga}_{1-x} \mathrm{~N} / \mathrm{Al}_{\mathrm{y}} \mathrm{Ga}_{1-y} \mathrm{~N}$ kuantum kuyusunun potansiyel profili üzerine kendiliğinden ve piezoelektrik polarizasyon alanlarının etkisini hesaba katarak sistemin optik düzeltmeleri üzerindeki etkisini tartışmışlar ve ayrıca bu makalede sistemin ikinci harmonik nesilleri üzerine de çalışmışlardır. Zeiri ve arkadaşları [19] rezonant güçlendirme için asimetrik $(\mathrm{CdS} / \mathrm{ZnSe})$ / X-BeTe kuantum kuyusunun doğrusal olmayan optik özelliklerini incelemiştir. Keshavarz ve Karimi [20], simetrik çift yarı parabolik kuantum kuyusundaki doğrusal ve doğrusal olmayan altbantlar arası optik soğurma katsayıları üzerinde çalışmışlardır. Rodriguez ve arkadaşları [21] n-katkılı bir GaAs kuantum kuyusunun kırılma indisi değişimi ve optik soğurma katsayılarını hesaplamıştır. Kasapoglu ve arkadaşları [22], rezonant olmayan yüksek frekanslı yoğun lazer alanı tarafından modüle edilen bir Morse kuantum kuyusundaki sığ donor safsızlıklarıyla ilgili bağlanma enerjisini ve optiksel soğurma katsayılarını araştırmıştır. Al ve arkadaşları [23], uygulanan bir statik elektrik alan altındaki Tietz-Hua kuantum kuyusundaki donor safsızlık durumlarının bağlanma enerjisini ve sistemin optik soğurma katsayılarını hesaplamışlardır. Kasapoglu ve arkadaşları [24], "12-6" ayarlı GaAs / GaAlAs çift kuantum kuyusundaki donor safsızlık durumlarının bağlanma enerjisi ve optik soğurma katsayıları üzerine uygulanan harici alanların etkisini incelemişlerdir.

$\mathrm{Bu}$ çalışmada, harici olarak uygulanan statik elektrik ve manyetik alanın Rosen-Morse kuyusunun merkezine yerleştirilmiş verici atomunun 1s safsızlık durumlarının bağlanma enerjileri üzerindeki etkisi araştırılmıştır. Ayrıca, donor safsızlıkları olan ve olmayan Rosen-Morse kuantum kuyusunun iletim bandındaki en düşük iki elektron durumu arasındaki geçişlere karşılık gelen doğrusal, doğrusal olmayan ve toplam optik soğurma katsayıları üzerine bu dış alanların etkisini de hesaplanmıştır. Etkin kütle ve parabolik bant yaklaşımı çerçevesinde yapının öz-enerjilerinin ve öz-fonksiyonlarının hesaplamaları, harici alanların yokluğunda sonsuz kuantum kuyusunun kesin çözümleri olarak elde edilen fonksiyonların seri ifadesi kullanılarak yapılmıştır. Daha sonra, safsızlık bağlama enerjisi, hidrojen benzeri deneme dalgası fonksiyonları kullanan bir varyasyonel yaklaşım metodu ile belirlenmiştir. Son olarak, sistemin doğrusal olmayan optik özellikleri, iki seviyeli bir yaklaşım dahilinde kompakt yoğunluklu matris formalizmi kullanılarak hesaplanmıştır. Makalenin organizasyonu aşağıdaki gibidir: teorik hesaplamalar Bölüm 2'de sunulmaktadır. Bölüm 3'de elde edilen sayısal sonuçlar tartışılmış ve son bölümde ise çalışmanın sonunda elde edilen fiziksel sonuçlar rapor halinde okuyucuya sunulmuştur. 


\section{MATERYAL VE METOT}

$\mathrm{Bu}$ makalede sunulan araştırma problemi, statik elektrik ve manyetik alan altındaki Rosen-Morse kuantum kuyusunun elektronik ve optiksel özellikleri üzerine z-doğrultusu boyunca yerleşmiş alan bir donor safsızlığının etkisini araştırmaktır. Burada, biz Rosen-Morse kuantum kuyusunun z-doğrultusunda büyütüldüğünü kabul ediyoruz. Ayrıca bu yapının büyütme doğrultusuna paralel uygulanan $F$ büyüklüğünde bir statik elektrik alanı $\overrightarrow{\boldsymbol{F}}=F \hat{z}$ ve büyütme doğrultusuna dik doğrultuda uygulanan statik bir manyetik alanı $\overrightarrow{\boldsymbol{B}}=B \hat{x}$ altında olduğunu göz önüne alıyoruz. Landau ayarındaki manyetik alanın karşıllk gelen vektör potansiyeli şu şekilde verilir: $\overrightarrow{\boldsymbol{A}}(\overrightarrow{\boldsymbol{r}})=-B z \hat{y}$. Bu şartlar altında ve etkin kütle yaklaşımından yararlanılarak, elektron-verici safsızlık çifti sistemi için Hamiltoniyen şu şekilde verilir:

$$
H=-\frac{1}{2 m^{*}}\left(\overrightarrow{\boldsymbol{p}}+\frac{e}{c} \overrightarrow{\boldsymbol{A}}(\overrightarrow{\boldsymbol{r}})\right)^{2}+V_{R M}(z)+e F z-\frac{e^{2}}{\varepsilon \sqrt{\rho^{2}+\left(z-z_{i}\right)^{2}}}
$$

Burada, $m^{*}$ elektronun etkin kütlesini, $\overrightarrow{\boldsymbol{p}}$ momentum operatörünü, $e$ elektron yükünü, $c$ boş uzaydaki ışı hızını ve $\rho=\sqrt{x^{2}+y^{2}} x-y$ düzlemindeki elektron-safsızlık arasındaki mesafeyi tanımlar. Ek olarak, $z$ ve $z_{i}$, sırasıyla Rosen-Morse kuantum kuyusu boyunca elektron ve safsızlık pozisyonlarıdır. Denklem (1) deki kuşatma potansiyeli $V_{R M}(z)$ ifadesi şu şekilde yazılabilir:

$$
V_{R M}(z)=-V_{1} \sec ^{2}(\eta z)+V_{2} \tanh ^{2}(\eta z) \text {. }
$$

Burada $V_{1}$ ve $V_{2}$ Rosen-Morse kuantum kuyusunun derinlik parametrelerini ve $\eta$ ise kuantum kuyusunun genişlik parametresidir.

Elektronik dalga fonksiyonlarını ve 1s benzeri durumun karşılık gelen enerjilerini elde etmek için, iyi bilinen varyasyon yöntemi kullanılır. Bu durumda, sistemi tanımlayan deneme dalgası fonksiyonu $\psi(\rho, z, \lambda)$, zarf dalga fonksiyonu $\varphi(z)$ ve hidrojenik benzeri bir dalga fonksiyonundan $\chi(\rho, z, \lambda)$ üretilir:

$$
\psi(\rho, z, \lambda)=\varphi(z) \chi(\rho, z, \lambda) .
$$

1s durumu için hidrojenik dalga fonksiyonları [23]

$$
\chi(\rho, z, \lambda)=N e^{\frac{\sqrt{\rho^{2}+\left(z-z_{i}\right)^{2}}}{\lambda}}
$$

ile verilir. Burada, $N$ normalizasyon sabitini ve $\lambda$ variyasyonel parametreyi temsil eder. Taban durum safsizlık bağlanma enerjisi

$$
E_{b}^{\lambda}=E_{z}-\min _{\lambda}\langle\psi|H| \psi\rangle
$$

ile verilir. Burada, $E_{z}$ Rosen-Morse kuantum kuyusunda kuşatılmış elektronun taban durum enerjisidir ve ikinci terim varyasyonel parametresine göre Denklem (1) 'de Hamiltoniyen'in beklenen değerini minimuma indirerek bulunan safsizlık enerjisi $E_{1 s}$ verir.

Kompakt yoğunluk matris formalizmi kullanılarak 1s benzeri durumlar için enerji öz değerleri ve karşllık gelen dalga fonksiyonları elde edildikten sonra, iki elektron durumu arasındaki doğrusal ve üçüncü dereceden doğrusal olmayan optik geçişlerdeki değişiklikler aşağıdaki denklemler kullanılarak hesaplanmıştır.

$$
\begin{gathered}
\beta^{(1)}(\omega)=\omega \sqrt{\frac{\mu}{\varepsilon}} \frac{\left|\mu_{12}\right|^{2} \sigma_{v} \hbar \Gamma_{12}}{\left(E_{21}-\hbar \omega\right)^{2}+\left(\hbar \Gamma_{12}\right)^{2}} \\
\beta^{(3)}(\omega, I)=-2 \omega \sqrt{\frac{\mu}{\varepsilon}}\left(\frac{I}{\varepsilon_{0} n_{r} c}\right) \frac{\left|\mu_{12}\right|^{4} \sigma_{v} \hbar \Gamma_{12}}{\left[\left(E_{21}-\hbar \omega\right)^{2}+\left(\hbar \Gamma_{12}\right)^{2}\right]^{2}} \times\left(1-\frac{\left|\mu_{22}-\mu_{11}\right|^{2}}{4\left|\mu_{12}\right|^{2}} \times \frac{\left\{\left(E_{21}-\hbar \omega\right)^{2}-\left(\hbar \Gamma_{12}\right)^{2}+2\left(E_{21}\right)\left(E_{21}-\hbar \omega\right)\right\}}{\left(E_{21}\right)^{2}+\left(\hbar \Gamma_{12}\right)^{2}}\right)
\end{gathered}
$$

Toplam optiksel soğurma katsayısı $\beta(\omega, I)$ Denklem (6) ile Denklem (7) de verilen iki terimin toplamından $\beta(\omega, I)=\beta^{(1)}(\omega)+\beta^{(3)}(\omega, I)$ elde edilir. Denklem (6) ve (7) ifadelerindeki terimlerin fiziksel tanımları şöyledir: $\mu_{i j}=\left\langle\psi_{i}|e z| \psi_{j}\right\rangle$ köşegen dışı matris elemanının mutlak değerini temsil eder, $\mu$ geçirgenlik, $n_{r}$ kırılma indisini, $\sigma_{v}$ sistemdeki elektron yoğunluğunu, $\omega$ sistemi uyaran fotonun açısal frekansını, $I$ gelen elektromanyetik alanın yoğunluğunu, $E_{21}=E_{2}-E_{1}$ farklı iki elektronik durumun enerji aralığını ve $\Gamma_{12}=\frac{1}{\tau_{12}}$ bant içi gevşeme zamanı göstermektedir. 
$\mathrm{Bu}$ çalışmada sayısal hesaplamalarda kullanılan fiziksel sabitler aşağıda verilmiştir:

$m^{*}=0.067 m_{0}$ (burada $m_{0}$ serbest elektron kütlesidir.), $e=1.602 \times 10^{-19} \mathrm{C}, \hbar=1.056 \times 10^{-34} \mathrm{Js}$, $\sigma_{v}=3 \times 10^{22} \mathrm{~m}^{-3}, n_{r}=3.2, \mu=4 \pi \times 10^{7} \mathrm{Hm}^{-1}, \varepsilon=12.58, \varepsilon_{0}=8.854 \times 10^{-12}, I=0.01 \mathrm{MW} / \mathrm{cm}^{2}, V_{1}=$ $V_{2}=114 \mathrm{meV}$ ve $\tau_{12}=0.2 \mathrm{ps}$.

\section{BULGULAR VE TARTIŞMA}

$\mathrm{Bu}$ çalışmada Rosen-Morse kuantum kuyusundaki temel durumla ilişkili hidrojenik donor safsızlığının bağlanma enerjisi üzerindeki harici statik elektrik ve manyetik alanın etkisi teorik olarak etkin kütle yaklaşımında varyasyonel bir yöntem kullanılarak hesaplanmıştır. Ayrıca, bu yapıdaki safsızlıklarla ilgili doğrusal olmayan optik özellikler, kompakt yoğunluklu matris yaklaşımı kullanılarak incelenmiştir.
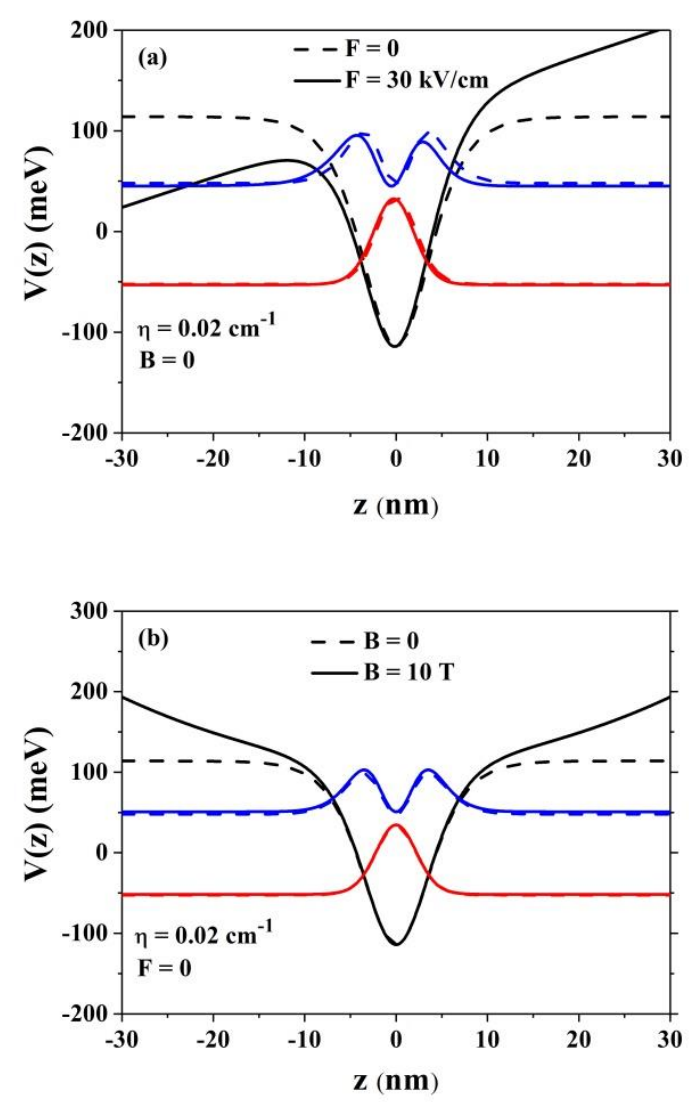

Şekil 1. (a) Statik elektrik alan ve (b) Statik manyetik alanın farklı değerleri için sistemin kuşatma potansiyel profilleri ve ilk iki altbant enerji seviyeleri ile bu enerji seviyelerine karşıllk gelen dalga fonksiyonlarının karesi

İlk olarak, Şekil 1 (a) ve 1 (b) 'de -şematik olarak- yapının kuşatma potansiyelini ve en düşük iki altbant enerji seviyesini ve bu enerji seviyelerine karşılık gelen olasılık yoğunluklarını gösteriyoruz. Bu şekiller, yapıya dışarıdan uygulanan alanların incelenen heteroyapının elektronik ve optik özellikleri üzerindeki etkisi hakkında bize önemli fikirler vermektedir. Sistematik bir karşılaştırma yapmak için öncelikle tüm dış alanları kapatıyoruz. Bu durumda, taban durum dalga fonksiyonunun tam olarak yapının ortasında bulunduğunu görüyoruz. Bu karakter, uygulanan alanlar yokken yapının simetrisi nedeniyle taban durumun güçlü bir şekilde kuantum sınırlandırılmasından kaynaklanmaktadır. Kuantum mekaniği ilkelerinden beklenebileceği gibi, en düşük durumlara karşılık gelen dalga fonksiyonları, uyarılmış olanlardan daha sınırlıdır. Sonuç olarak, uzaysal yayılma, yüksek enerji seviyeler için alt enerji seviyelerden daha önemlidir. Bu şekillerden açıkça görüldüğü gibi birinci 
uyarılmış seviyeye karşılık gelen olasılık yoğunluğunun uzaysal dağılımının yapının ortasında iki ana zirveye sahip olduğu fark edilebilir. Bununla birlikte, ikinci uyarılmış dalga fonksiyonu daha fazla yayılmıştır.

Şekil 1 (a), statik elektrik alanın elektronik durumlar üzerindeki etkisini göstermektedir. Burada biz, elektrik alanını $30 \mathrm{kV} / \mathrm{cm}$ değerinde açıp manyetik alanı sıfirda tutuyoruz. Yapının büyütme doğrultusuna paralel uygulanan statik elektrik alanın etkisi ile Rosen-Morse kuantum kuyusundaki kuşatılmış elektronlar, elektrik alanına ters yönde etki eden kuvvetle yapının sol tarafına hareket ederek kuşatma potansiyelini büker. Böylece yapının simetrisi bozulur ve taban durum ile ilk uyarılmış durum enerji seviyesi arasındaki enerji farkı azalır. Ek olarak, bu enerji seviyeleri uzayın farklı yerlerine yerleşir ve sonuç olarak taban ile ilk uyarılmış durum dalga fonksiyonları arasındaki örtüşme azalır.

Sisteme statik bir manyetik alanın etkisi Şekil 1 (b) 'de gösterilmektedir. Bu durumda statik manyetik alan 10 T'de sabitlenir. Diğer alanlar kapatılır. Bilindiği gibi, statik manyetik alan parabolik bir sınırlama getirir. Bu sınırlandırmanın etkisiyle, bağlı durumlar kuantum kuyusunun tabanına yaklaşır ve en düşük iki enerji seviyesi arasındaki enerji farkı da artar. Ayrıca, taban durum ve ilk uyarılmış durum enerji seviyeleri dalga fonksiyonları, artan statik manyetik alanla daha fazla örtüşür.
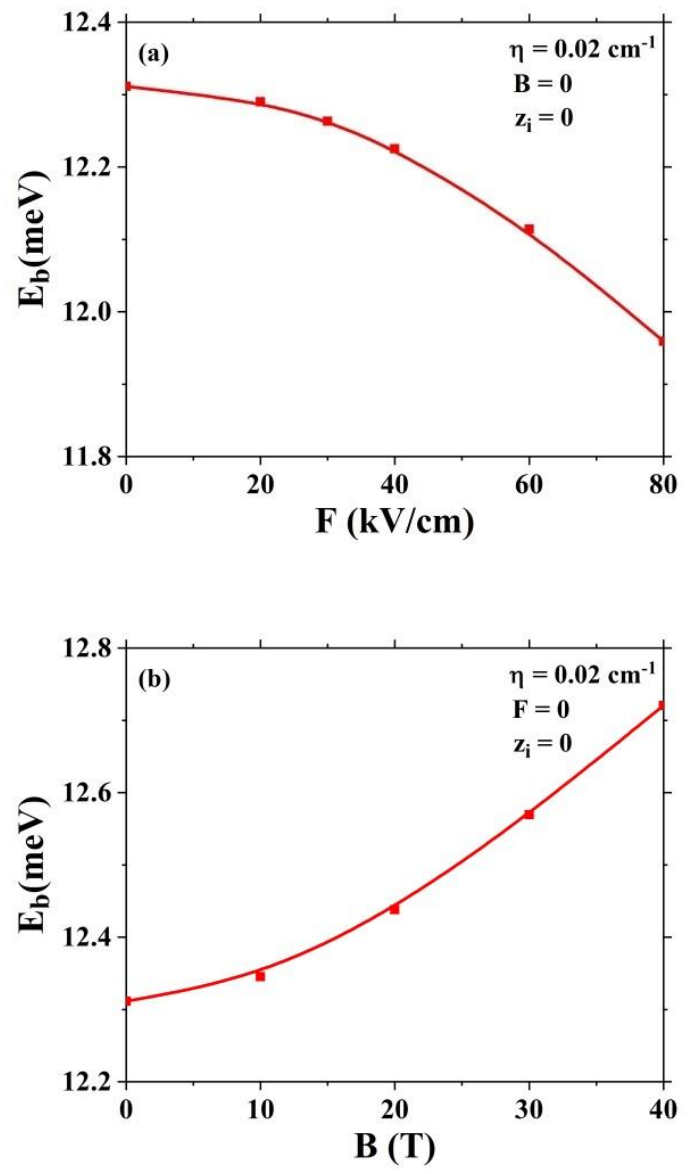

Şekil 2. (a) Statik elektrik alanın ve (b) Statik manyetik alanın bir fonksiyonu olarak yapının merkezindeki bir safsızlık atomunun bağlanma enerjisinin değişimi

Şekil 2 (a), Rosen-Morse kuantum kuyusundaki safsızlık atomunun bağlanma enerjisinin statik elektrik alanına göre değişimini göstermektedir. Bu şekilden de görüldüğü gibi statik elektrik alanın artmasıyla safsızlık bağlanma enerjisi azalır. Ayrıca yapı üzerine uygulanan statik elektrik alanın artması ile yük taşıyıcılarının (elektron-safsızlık atomu) geometrik kuşatılması azalır. Geometrik kuşatmadaki bu azalma, aynı düzlemde 
elektron ve safsızlık atomunun bulunma olasılığını azaltır. Böylece, elektron ve safsızlık atomu arasındaki Coulombik etkileşim azaldığı için, safsızlık bağlanma enerjisi de azalır.

Şekil 2b'de, Rosen-Morse kuantum kuyusundaki safsızlık atomu bağlama enerjisinin statik manyetik alana göre değiş̧imi verilmiştir. Bu şekilden de açıkça görüldüğü üzere statik manyetik alanın büyüklüğündeki artışla birlikte, donor safsızlık atomunun bağlama enerjisi artar. Bilindiği üzere yük taşıyıcılarının geometrik kuşatılması (elektron-safsızlık atomu), manyetik alanın artmasıyla artar (bkz. Şekil 1 (b)). Geometrik kuşatmadaki bu artış, elektron ve safsızlık atomunun aynı düzlem içinde bulunma olasılığını artırır. Bu davranış, elektron ve safsızlık atomu arasındaki Coulombic etkileşimini arttırdığından, safsızlık bağlanma enerjisi de artar.
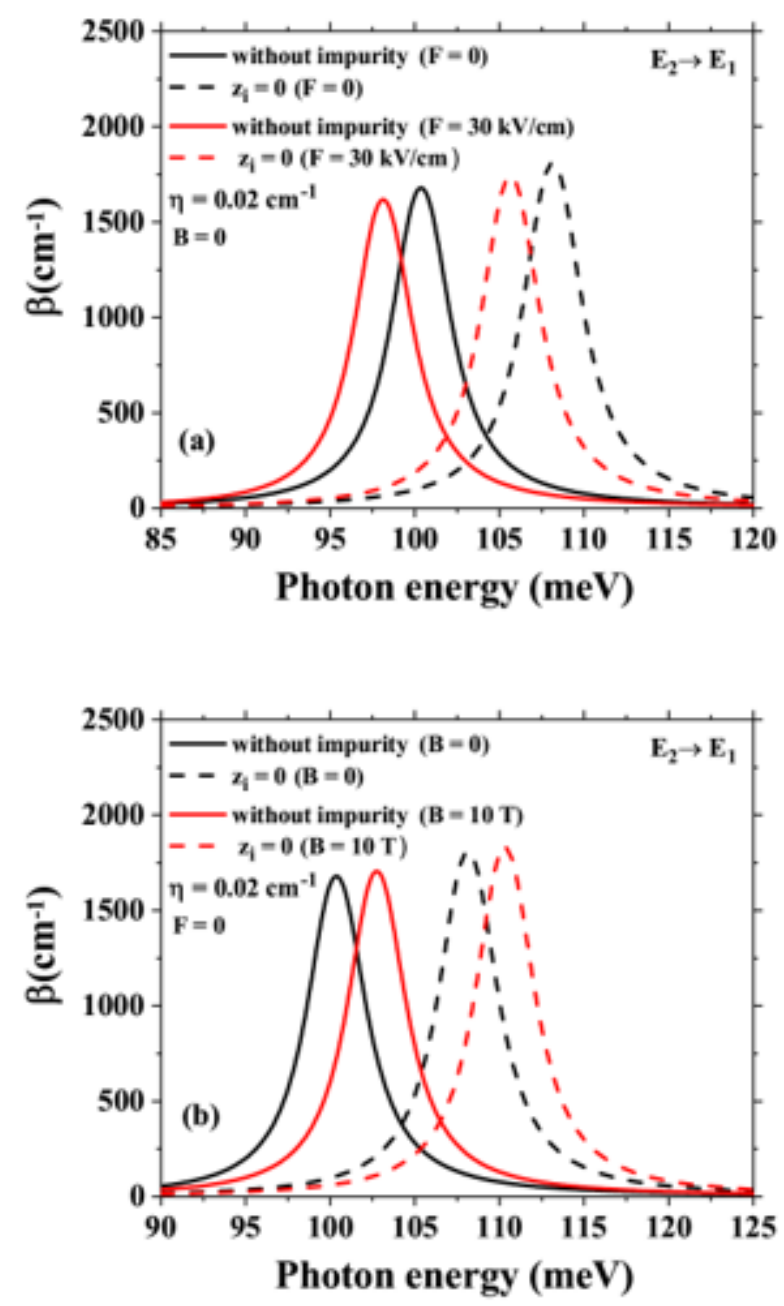

Şekil 3. İki farklı (a) Statik elektrik alan ve (b) Statik manyetik alan değerleri için gelen foton enerjisine karşı toplam soğurma katsayısı

Şekil 3 (a) 'da biz daha önce bahsettiğimiz en düşük iki elektron enerji seviyesi arasındaki geçişler ile alakalı Toplam Optik Soğurma Katsayısı (TOAC) spektrumunu gelen foton enerjisinin bir fonksiyonu olarak sunuyoruz. Bu şekildeki düz çizgiler $F=0$ ve kesikli çizgiler $(F=30 \mathrm{kV} / \mathrm{cm})$ etkisini göstermektedir. TOAC spektrumu, ilgili elektron seviyeleri arasındaki enerji farkının azalmasına bağlı olarak azalan genliklerle daha düşük foton enerjilerine doğru kayar. Statik elektrik alanı, dipol moment matris elemanlarının azalmasına neden olur. Sisteme katılan donor safsızlık atomu, sistemin bağlı durum enerji spektrumunu değişstirir. Safsızlık atomu, alt bantlar arası geçişlerin meydana geldiği en düşük iki enerji seviyesi arasındaki enerji farkını büyütür. Böylece, yapıya katılan merkezi safsızık atomu en düşük iki elektron enerji seviyesi arasındaki enerji farkını artırdığı için TOAC'lar daha yüksek enerjilere doğru kayar. 
Statik manyetik alanın sistemin elektronik ve optik özellikleri üzerinde önemli bir etkiye sahip olduğu iddiasını desteklemek için, Şekil 3 (b) 'de iki farklı manyetik alan için gelen foton enerjisinin bir fonksiyonu olarak TOAC'ların değișimini gösteriyoruz. Bunun için önce sisteme uygulanan statik elektrik alan kapatılır. Şekil 3 (b) 'yi inceleyerek, TOAC'ların genliklerinin artan statik manyetik alanı ile büyüdüğünü görebiliriz. Ek olarak, statik manyetik alanın artmasıyla TOAC piklerinin daha yüksek enerjilere doğru hareket ettiğini yani mavi renge doğru bir kaymanın olduğu açıkça görülebilir. Statik elektrik alanındaki duruma benzer şekilde, donor safsızlık atomu, sistemin toplam enerjisini bağlanma enerjisinin değeri kadar arttırır. Bu artan enerji farkı, sistemin TOAC'larının tepe noktasının daha yüksek enerjilere doğru kaymasına neden olur.

\section{SONUÇ VE ÖNERILER}

Özet olarak, bu çalışmada Rosen-Morse kuantum kuyusuna dayalı bir heteroyapıdaki toplam optik soğurma katsayılarının yanı sıra donor safsızlık bağlama enerjilerinin sayısal bir araştırmasını gerçekleştirdik. Bu yapı statik elektrik ve manyetik alan etkisi altında incelenmiştir. Etkin kütle ve zarf dalga fonksiyonu yaklaşımları çerçevesinde sistemin alt bant enerji seviyeleri ve zarf dalga fonksiyonları hesaplanmış ve daha sonra varyasyonel yöntem kullanılarak safsızlık bağlanma enerjisi elde edilmiştir. Elde edilen sonuçlar donor safsızlık bağlama enerjisinin, bu yapı için harici olarak uygulanan statik elektrik ve manyetik alana zayıf bir şekilde bağlı olduğunu göstermiştir. Safsızlık bağlama enerjisi, temel olarak Rosen-Morse kuantum kuyusundaki kuşatılmış elektronun elektronik dalga fonksiyonunun uzaysal dağılımı tarafından belirlenen karakteristik bir özellik sunar. Ayrıca elde edilen sayısal sonuçlar, sistemin toplam optik soğurma katsayılarının, statik elektrik alan ile kırmızıya (düşük enerji seviyelerine) ve statik manyetik alan ile de maviye bir kayma sergilediğini göstermektedir. Elde edilen bu sonuçların yeni nesil elektronik ve optoelektronik cihazların tasarımı ve üretimi ile ilgilenenler için faydalı olabileceğine inanıyoruz.

\section{KAYNAKLAR}

[1] Hien N. D., Dinh L., \& Anh N. T. T. (2020). Influence of confined optical phonons on the magneto-optical properties in parabolic quantum wells, Journal of Physics and Chemistry of Solids, 145, 109501.

[2] Tung L. V., Vinh P. T., \& Phuc H. V. (2018). Magneto-optical properties of semi-parabolic plus semi-inverse squared quantum wells, Physica B: Condensed Matter, 539, 117-122.

[3] Ungan F., Restrepo R. L., Mora-Ramos M. E., Morales A. L., \& Duque C. A. (2014). Intersubband optical absorption coefficients and refractive index changes in a graded quantum well under intense laser field: Effects of hydrostatic pressure, temperature and electric field, Physica B: Condensed Matter, 434, 26-31.

[4] Dakhlaoui H., (2018).Tunability of the optical absorption and refractive index changes in step-like and parabolic quantum wells under external electric field, Optik, 168, 416-423.

[5] Niculescu E. C., Radu A., \& Stefe M. (2009). Laser effects on the donor states in V-shaped and inverse Vshaped quantum wells, Superlattices and Microstructures, 46, 443-450.

[6] Şakiroğlu S., Ungan F., Yesilgul U., Mora-Ramos M. E., Duque C. A., Kasapoglu E., Sari H., \& Sökmen I., (2012). Non linear optical rectification and the second and third harmonic generation in Pöschl-Teller quantumwell under the intense laser field, Physics Letters A, 376, 1875-1880.

[7] Zhang Z. H., Zou L., Guo K. X., \& Yuan J. H., (2016). The nonlinear optical rectification in asymmetrical and symmetrical Gaussian potential quantum wells with applied electric field, Optics Communications, 359, 316-321.

[8] Restrepo R. L., Morales A. L., Akimov V., Tulupenko V., Kasapoglu E., Ungan F., \& Duque C. A., (2015). Intense laser field effects on a Woods-Saxon potential quantum well, Superlattices and Microstructures, 87, 143-148.

[9] Mo S., Guo K., Lui G., He X., Len J., \& Zhou Z. (2020). Exciton effect on the linear and nonlinear optical absorption coefficients and refractive index changes in Morse quantum wells with an external electric field, Thin Solid Films, 710, 138286.

[10] Ungan F., Sari H., Kasapoglu E., Yesilgul U., Sakiroglu S., \& Sökmen I., (2018). Study of electron-related optical responses in the Tietz-Hua quantum well: Role of applied external fields, Photonics and Nanostructures - Fundamentals and Applications, 32, 47-52.

[11] Ungan F., \& Bahar M. K., (2019). Optical specifications of laser-induced Rosen-Morse quantum well, Optical Materials, 90, 231-237.

[12] Nakamura S., Senoh M. \& Mukai T., (1993). P-GaN/N-InGaN/N-GaN Double-Heterostructure Blue-LightEmitting Diodes, Japon Journal of Applied Physics, 32, 1A/B.

[13] Nguyen L. D., Brown A. S., Thompson M.A. \& Jelloian L.M., (1992). 50-nm self-aligned-gate pseudo morphic AlInAs/GaInAs high electron mobility transistors, IEEE Transactions on Electron Devices, 39, 9. 
[14] Ejeckam F. E., Chua C. L., Zhu Z. H., \& Lo Y. H., (1995). High-performance InGaAs photodetectors on Si and GaAs substrates, Applied Physics Letters, 67, 26.

[15] Zheng J., Zhang Y., Li L., Tang S., Sh Y. \& Chen X., (1992). Temperature dependence of GaAs-AlGaAs vertical cavity surface emitting lasers, Applied Physics Letters, 60, 6.

[16] Shi J.\& Pan S. (1995). Calculation of linear and non linear intersubband optical absorptions in a superlattice with a step-well basis, Superlattice sand Microstructures, 17, 91-95.

[17] Radu A. (2013) Laser-dressing of electronic quantum states in graded semiconductor nanostructures, Solid State Communications, 157, 11-15.

[18] Karimi M. J. \& Vafaei H. (2015). Second-order nonlinear optical properties in a strained InGaN/AlGaN quantum well under the intense laser field", Superlattices and Microstructrures, 78, 1-11.

[19] Zeiri N., Sfina N., Nasrallah S. A. B. \& Said M., (2013). Intersubband resonant enhancement of the non linear optical properties in asymmetric $(\mathrm{CdS} / \mathrm{ZnSe}) / \mathrm{X}-\mathrm{BeTe}$ based quantum wells, Optical Materials, 35, 875-880.

[20] Keshavarz A. \& Karimi M. (2010). Linear and nonlinear intersubband optical absorption in symmetric double semi-parabolic quantumwells", Physics Letters A, 374, 2675-2680.

[21] Rodriguez-Magdaleno K., Martinez-Orozco J., Rodriguez-Vargas I., Mora-Ramos M. \& Duque C. A. (2014). Asymmetric GaAs n-type double $\delta$-doped quantum wells as a source of intersubband-related nonlinear optical response: Effects of an applied electric field, Journal of Luminescence, 147, 77-84.

[22] Kasapoglu E., Sakiroglu S., Sari H., Sökmen I., \& Duque C. A. (2019). Morse quantum well modulated by nonresonant intense laser field: Binding energy and optical absorption related to shallow donor impurities, Optik, 181, 432-439.

[23] Al E. B., Kasapoglu E., Sakiroglu S., Duque C. A., \& Sökmen I. (2018). Binding energy of donor impurity states and optical absorption in the Tietz-Hua quantum well under an applied electric field, Journal of Molecular Structure, 1157, 288-291.

[24] Kasapoglu E., Sakiroglu S., Sari H., Sökmen I. \& Duque C. A. (2019). Binding energy and optical absorption of donor impurity states in "12-6" tuned GaAs/GaAlAs double quantum well under the external fields, Physica B: Condensed Matter, 554, 72-78. 